\title{
Common variation in oxidative phosphorylation genes is not a major cause of insulin resistance or type 2 diabetes
}

\author{
L. S. Snogdal • M. Wod • N. Grarup • M. Vestmar • T. Sparsø • T. Jørgensen • \\ T. Lauritzen $\cdot$ H. Beck-Nielsen • J. E. Henriksen • O. Pedersen • T. Hansen • K. Højlund
}

Received: 9 September 2011 / Accepted: 25 October 2011 /Published online: 18 November 2011

(C) Springer-Verlag 2011

\begin{abstract}
Aims/hypothesis There is substantial evidence that mitochondrial dysfunction is linked to insulin resistance and is present in several tissues relevant to the pathogenesis of type 2 diabetes. Here, we examined whether common variation in genes involved in oxidative phosphorylation (OxPhos) contributes to type 2 diabetes susceptibility or influences diabetes-related metabolic traits.

Methods OxPhos gene variants $(n=10)$ that had been nominally associated $(p<0.01)$ with type 2 diabetes in a recent genome-wide meta-analysis $(n=10,108)$ were selected for follow-up in 3,599 type 2 diabetic and 4,956 glucosetolerant Danish individuals. A meta-analysis of these variants was performed in 11,729 type 2 diabetic patients and 43,943 non-diabetic individuals. The impact on OGTT-derived metabolic traits was evaluated in 5,869 treatment-naive individuals from the Danish Inter99 study.
\end{abstract}

Electronic supplementary material The online version of this article (doi:10.1007/s00125-011-2377-0) contains peer-reviewed but unedited supplementary material, which is available to authorised users.

L. S. Snogdal • M. Wod • H. Beck-Nielsen · J. E. Henriksen •

T. Hansen $\cdot$ K. Højlund $(\bowtie)$

Diabetes Research Centre, Department of Endocrinology,

Odense University Hospital,

Kløvervænget 6, 4th Floor,

5000 Odense, Denmark

e-mail: k.hojlund@dadlnet.dk

L. S. Snogdal • M. Wod • H. Beck-Nielsen · J. E. Henriksen •

K. Højlund

Institute of Clinical Research, University of Southern Denmark,

Odense, Denmark

N. Grarup $\cdot$ M. Vestmar $\cdot$ T. Spars $\varnothing \cdot$ O. Pedersen $\cdot$ T. Hansen The Novo Nordisk Foundation Center for Basic Metabolic Research, Faculty of Health Sciences, University of Copenhagen, Copenhagen, Denmark
Results The minor alleles of COX10 rs9915302 $(p=0.02)$ and $C O X 5 B$ rs $1466100(p=0.005)$ showed nominal association with type 2 diabetes in our Danish cohort. However, in the meta-analysis, none of the investigated variants showed a robust association with type 2 diabetes after correction for multiple testing. Among the alleles potentially associated with type 2 diabetes, none negatively influenced surrogate markers of insulin sensitivity in non-diabetic participants, while the minor alleles of UQCRC1 rs2228561 and COX10 rs10521253 showed a weak $(p<0.01$ to $p<0.05)$ negative influence on indices of glucose-stimulated insulin secretion.

Conclusions/interpretation We cannot rule out the possibility that common variants in or near OxPhos genes may influence beta cell function in non-diabetic individuals. However, our quantitative trait studies and a sufficiently large meta-analysis indicate that common variation in

\author{
T. Jørgensen \\ Research Centre for Prevention and Health, \\ Glostrup University Hospital, \\ Glostrup, Denmark \\ T. Jørgensen $\cdot$ O. Pedersen \\ Faculty of Health Sciences, University of Copenhagen, \\ Copenhagen, Denmark \\ T. Lauritzen \\ Department of General Practice, Faculty of Health Sciences, \\ University of Aarhus, \\ Aarhus, Denmark \\ O. Pedersen \\ Hagedorn Research Institute, \\ Gentofte, Denmark
}


proximity to the examined OxPhos genes is not a major cause of insulin resistance or type 2 diabetes.

Keywords Genetics · Human · Meta-analysis · Oxidative phosphorylation - Type 2 diabetes

\begin{tabular}{|c|c|}
\hline \multicolumn{2}{|c|}{ Abbreviations } \\
\hline ADDITION & Anglo-Danish-Dutch Study of Intensive \\
\hline & Treatment In PeOple With screeN \\
\hline & Detected Diabetes in Primary Care \\
\hline AIR & Acute insulin response \\
\hline BIGTT-S $_{\mathrm{I}}$ & BIGTT sensitivity index \\
\hline CIR & Corrected insulin response \\
\hline $\mathrm{COX}$ & Cytochrome $c$ oxidase \\
\hline DGI & Diabetes Genetic Initiative \\
\hline DI & Disposition index \\
\hline DIAGRAM & $\begin{array}{l}\text { Diabetes Genetics Replication and } \\
\text { Meta-analysis }\end{array}$ \\
\hline ETC & Electron transport chain \\
\hline GWA & Genome-wide association \\
\hline HOMA-IR & HOMA of insulin resistance \\
\hline ISI & Insulin sensitivity index \\
\hline MAF & Minor allele frequency \\
\hline OxPhos & Oxidative phosphorylation \\
\hline SNP & Single-nucleotide polymorphism \\
\hline
\end{tabular}

\section{Introduction}

Type 2 diabetes is a heterogeneous disease caused by a complex interplay between numerous genetic, epigenetic and environmental factors. Insulin resistance in skeletal muscle, adipose tissue and liver, together with failure of the pancreatic beta cells to compensate for this abnormality, plays a major role in the pathogenesis of type 2 diabetes. Skeletal muscle, which accounts for the majority of insulinstimulated glucose uptake, is a major site of insulin resistance in type 2 diabetic and high-risk individuals [1]. Numerous investigations have demonstrated that skeletal muscle of insulin-resistant individuals shows decreased insulin action on glucose transport and glycogen synthesis, accumulation of lipid metabolites and impaired insulin signalling through proximal effector proteins and glycogen synthase [1-6]. Recent studies have provided strong evidence of a link between insulin resistance and mitochondrial dysfunction in human skeletal muscle [7]. Global approaches such as transcriptional profiling and proteomics have demonstrated a co-ordinated downregulation of mRNA levels and protein content of nuclearencoded genes involved in oxidative phosphorylation (OxPhos) in muscle of patients with type 2 diabetes and of high-risk individuals [8-11]. Moreover, a reduced content and impaired functional capacity of muscle mitochondria may also contribute to mitochondrial dysfunction in type 2 diabetes and obesity [12-14]. It has been proposed that mitochondrial dysfunction contributes to insulin resistance in skeletal muscle by causing an accumulation of lipid metabolites, which in turn may impair insulin signalling to glucose transport and glycogen synthesis [7, 15]. Evidence of mitochondrial dysfunction in other tissues relevant to the pathogenesis of type 2 diabetes is also emerging. This includes reports of reduced expression of electron transport chain (ETC) genes in adipose tissue of women with type 2 diabetes [16], and of impaired mitochondrial respiratory capacity in heart muscle [17] and liver from type 2 diabetic individuals [18]. Finally, mitochondrial ATP production is known to play a critical regulatory role in glucosestimulated insulin secretion, and several recent studies have suggested that defects in mitochondrial OxPhos could contribute to beta cell dysfunction in patients with type 2 diabetes [19-22].

Taken together, these findings support the hypothesis that mitochondrial dysfunction could be a unifying defect in the pathogenesis of type 2 diabetes [15]. However, it remains to be established whether mitochondrial dysfunction is a cause or consequence of insulin resistance and/or beta cell dysfunction in type 2 diabetes. To date, genome-wide association (GWA) studies have shown 38 common genetic variants to be robustly associated with type 2 diabetes, with 16 additional loci associated with traits of glucose homeostasis [23]. Together, they explain approximately $10 \%$ of type 2 diabetes heritability. Most of these risk alleles are located in genes believed to influence beta cell function [23], while only a few variants seem to be associated with insulin resistance, e.g. a variant upstream of IRSI [24]. Moreover, none of the above risk variants includes common variants in OxPhos genes. However, some studies have reported associations of a few specific variants in OxPhos genes (NDUFB6, COX7A1 and $A T P 5 O$ ) with certain phenotypical characteristics of type 2 diabetes and obesity [25-27].

Based on the accumulating evidence of mitochondrial dysfunction in tissues relevant to the pathogenesis of type 2 diabetes, the present study was performed to examine whether common variants in the nuclear-encoded genes, comprising the respiratory complexes $\mathrm{I}$ to $\mathrm{V}$ and related OxPhos genes, are associated with type 2 diabetes. Data from a genome-wide meta-analysis were explored [28] to select common OxPhos gene variants for follow-up in an independent cohort of Danish individuals. We also investigated the potential association of these genetic variants with well-defined quantitative traits of insulin sensitivity and beta cell function in a large Danish cohort of individuals without diabetes. 


\section{Methods}

Gene and single-nucleotide polymorphism selection We investigated the potential association between common variants in 100 nuclear-encoded OxPhos genes, representing the five respiratory complexes and related OxPhos proteins [29], and type 2 diabetes (Electronic supplementary material [ESM] Table 1). First, we explored the results from a metaanalysis of data from three GWA studies of 4,539 type 2 diabetic cases and 5,569 controls published by the Diabetes Genetics Replication and Meta-analysis (DIAGRAM) consortium [28] (www.well.ox.ac.uk/DIAGRAM/, accessed 1 July 2009). We found 2,542 imputed and genotyped singlenucleotide polymorphisms (SNPs) within or near 90 OxPhos genes (defined as boundary of coding region $\pm 10 \mathrm{~kb}$ ) (Fig. 1). For further evaluation, we then selected all 39 SNPs in the eight genes that showed nominal association $(p<0.01)$ with type 2 diabetes in the DIAGRAM study (ESM Table 2). When SNPs in high linkage disequilibrium $\left(r^{2}>0.8\right)$ in HapMap (Version Data Release 21a/phase II Jan07; www.hapmap.org, accessed 1 July 2009) were excluded, 11 SNPs in seven genes were found to cover all variation and were selected for genotyping in an independent replication cohort. We successfully genotyped ten SNPs in or near six OxPhos genes (NDUFV3, UQCRC1, COX4I1, COX5B, COX6B1 and COX10) in 9,745 Danish individuals. All further analyses are based on the evaluation of these SNPs (ESM Table 3).

Study population The case-control replication study of Danish individuals ( $n=3,599$ cases, $n=4,956$ controls) included all unrelated type 2 diabetic patients $(n=328)$ and glucose-tolerant controls $(n=4,444)$ from the Inter99 Study sample (ClinicalTrials.gov ID: NCT00289237) [30, 31], as well as type 2 diabetic patients $(n=1,577)$ from the Danish part of the Anglo-Danish-Dutch Study of Intensive Treatment In PeOple With screeN Detected Diabetes in Primary Care (ADDITION) intervention study (ClinicalTrials.gov ID: NCT00237549) [32] and individuals recruited from the outpatient clinic at Steno Diabetes Center (Gentofte, Denmark) ( $n=1,694$ cases, $n=512$ controls). Normal glucose tolerance and type 2 diabetes were defined according to World Health Organization 1999 criteria [33]. All type 2 diabetic patients were diagnosed by two independent plasma glucose measurements. Clinical characteristics of the three study groups and a brief description of the Inter99 and ADDITION studies are given in ESM Study Population and ESM Table 4. Participants of the Inter99 Study were investigated by an OGTT with measurements of plasma glucose and serum insulin at fasting, and at 30 and $120 \mathrm{~min}$ after glucose intake as described [34]. Analyses of these biochemical variables were used for studies of quantitative diabetes-related traits in 5,869 treatment-naive participants from the Inter99 cohort, excluding patients with previously diagnosed diabetes. Informed written consent was obtained from all participants before participation. The studies were approved by the local ethics committees of Copenhagen and Aarhus Counties and were conducted in accordance with the Helsinki Declaration II.

Derived estimates of insulin release and insulin sensitivity from an OGTT Indices of oral glucose-stimulated insulin

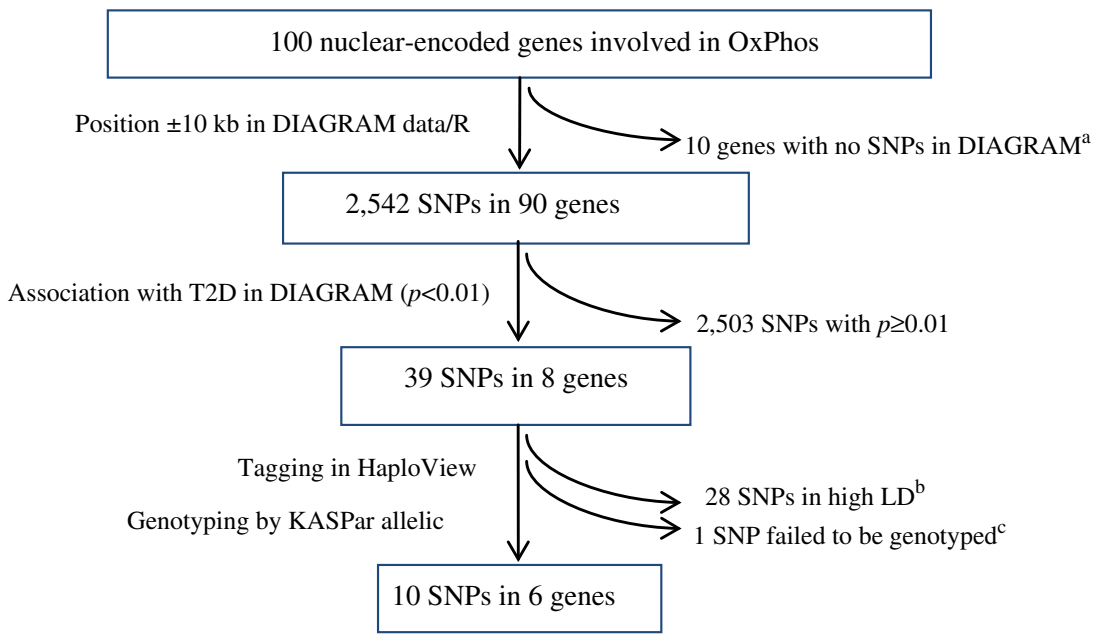

Fig. 1 Flowchart of the gene and SNP selection process. SNPs in or near 100 nuclear-encoded candidate genes involved in OxPhos were explored for potential association with type 2 diabetes (T2D) in the DIAGRAM study [28]. Eleven tag-SNPs in seven genes covered all variation in 39 SNPs showing nominal association with type 2 diabetes $(p<0.01)$. Ten SNPs in six genes were successfully genotyped for follow-up in a cohort of Danish individuals. ${ }^{\text {a }}$ For ten nuclear-encoded genes no SNPs were imputed or genotyped in DIAGRAM. ${ }^{\mathrm{b}}$ The $C O X 15 \mathrm{SNP}$ was in linkage disequilibrium (LD) with several of the COX10 SNPs. ${ }^{\text {CThe }}$ SDHA rs3756718 SNP failed to be genotyped and was excluded from further analysis 
secretion are reported as: (1) the insulinogenic index, calculated as (serum insulin $_{30 \mathrm{~min}}-$ serum $_{\text {insulin }}$ min $[\mathrm{pmol} / 1]) /($ plasma glucose $30 \min [\mathrm{mmol} / 1]) ;(2)$ the corrected insulin response (CIR), calculated as $(100 \times$ serum

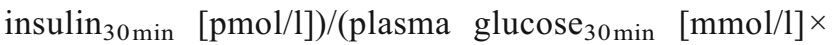
[plasma glucose $30 \mathrm{~min}-3.89]$ ); and (3) the BIGTT acute insulin response (AIR) index. The surrogate measures of insulin sensitivity are reported as: (1) the HOMA of insulin resistance (HOMA-IR), calculated as (plasma glucose ${ }_{0}$ min $[\mathrm{mmol} / \mathrm{l}] \times$ serum insulin $\left._{0 \mathrm{~min}}[\mathrm{pmol} / 1]\right) / 22.5$ [35]; (2) insulin sensitivity index (ISI), calculated as the reciprocal of HOMA-IR; and (3) the BIGTT sensitivity index $\left(\mathrm{S}_{\mathrm{I}}\right)$. The BIGTT indices, which apply information on sex and BMI, combined with plasma glucose and serum insulin during an OGTT, were calculated as reported [36]. Beta cell function was also assessed as the disposition index (DI), i.e. insulin secretion adjusted for insulin sensitivity by multiplying CIR and ISI, since these measures are not intrinsically interdependent.

Biochemical and anthropometrical measures Body weight and height were measured in light indoor clothing and without shoes. Blood samples were drawn after a $12 \mathrm{~h}$ overnight fast. Serum insulin levels were measured by a kit (AutoDELFIA; Perkin-Elmer, Wallac, Turku, Finland). Plasma glucose was measured using a glucose oxidase method (Granutest; Merck, Darmstadt, Germany).

Genotyping Genotyping of the ten genetic variants was performed using an allelic discrimination method (KASPar; Kbioscience, Hoddeston, UK). Success rates for all variants were above $96 \%$. Error rates were below $0.5 \%$. The minor allele frequencies (MAFs) were between 0.016 and 0.497 . All ten selected SNPs obeyed Hardy-Weinberg equilibrium $(p>0.05)$.

Statistical analysis In our case-control replication study of 3,599 patients with type 2 diabetes and 4,956 glucosetolerant controls, logistic regression with adjustment for age, sex and BMI assuming an additive model was applied to examine differences in genotype distributions between affected and unaffected participants. The quantitative trait analyses were performed in the Inter99 Study involving 5,869 treatment-naive participants and applying an additive general linear model adjusted for sex, age and BMI. All statistical analyses were performed using RGui version 2.9.0 (available at www.r-project.org/). A value of $p<0.05$ was considered statistically significant for nominal association. A meta-analysis of the selected ten SNPs in or near six OxPhos genes was also performed in a fixed-effect, inverse variance-weighted analysis combining ORs and 95\% CIs from our replication study of Danish individuals and results from a recent genome-wide meta-analysis combining data from eight studies published by the DIAGRAM consortium, hereafter designated DIAGRAM + [37]. It should be noted that the selection and genotyping of OxPhos SNPs in the Danish individuals were performed before publication of DIAGRAM + , and that the results from the latter are not publicly available. Homogeneity between studies was evaluated by $I^{2}$ and $Q$ test statistics. The meta-analyses were performed in $\mathrm{R}$ applying the 'meta' package and using an additive model. In the meta-analysis and quantitative trait analysis, $p$ values below a Bonferronicorrected (ten SNPs) threshold of $0.05 / 10=0.005$ were considered statistically significant.

Power calculations In the selection of SNPs for genotyping based on DIAGRAM results (4,539 type 2 diabetic cases, 5,569 controls), we had more than $80 \%$ statistical power to identify a SNP with an MAF of $40 \%$ and OR of 1.1 , and a SNP with an MAF of $5 \%$ and an OR of 1.25 at a significance level of 0.01 . Statistical power in case-control meta-analysis of the 11,729 type 2 diabetic patients and 43,943 non-diabetic individuals was calculated by simulations. The power calculations assumed a Bonferroniadjusted alpha-value of $0.05 / 10=0.005$ and a disease prevalence of $8 \%$. Given the MAFs for the ten investigated variants $(0.016$ to 0.497$)$, the meta-analysis had $80 \%$ statistical power to detect an OR of 1.05 in a case-control design for the most common variants (MAF 50\%), whereas we had $80 \%$ power to detect an OR of 1.20 for the lower frequency variants (MAF 1.6\%). Statistical power in studies of quantitative traits in the 5,869 Inter99 participants was estimated by simulations as described [34], showing that we had $80 \%$ statistical power to detect an effect equal to 0.27 SDs in the event of an MAF of $1.6 \%$ and an effect equal to 0.067 SDs for SNPs with an MAF of $50 \%$. In Inter99, this is equal to allelic differences in fasting plasma glucose of 0.22 and $0.055 \mathrm{mmol} / \mathrm{l}$, respectively.

\section{Results}

To find common variants in OxPhos genes potentially associated with type 2 diabetes, we examined data from a genome-wide meta-analysis published online by the DIAGRAM consortium [28]. A total of 39 SNPs in eight genes showed nominal associations $(p<0.01)$ with type 2 diabetes (Fig. 1, ESM Table 2). Interestingly, 35 of these SNPs were found in or near genes representing cytochrome $c$ oxidase (COX), i.e. complex IV of the ETC, including COX4I1, COX5B, COX6B1, COX10 and COX15. Of 25 SNPs localised to COX10, 21 showed a stronger nominal association $(p<0.001)$ with type 2 diabetes than any of the 14 SNPs in the other genes. We 
found that 11 SNPs in seven genes covered all variation and selected them for follow-up in an independent cohort.

We successfully genotyped ten SNPs in or near six OxPhos genes and examined them for association with type 2 diabetes in a case-control replication study of Danish individuals. In a model adjusted for age, sex and BMI, the nominal association with type 2 diabetes was replicated for the minor $\mathrm{G}$ allele of COX10 rs9915302 (OR 1.14 [95\% CI 1.02, 1.28]; $p=0.020$ ) (Table 1). In addition, the minor $\mathrm{T}$ allele of COX5B rs1466100 was nominally associated with type 2 diabetes (OR 1.62 [95\% CI 1.16, 2.28]; $p=0.0052$ ) in our Danish case-control study. This is in contrast to the DIAGRAM Study [28], where the major A allele of rs1466100 showed nominal association with type 2 diabetes (ESM Table 2). None of the other eight SNPs showed association with type 2 diabetes in our Danish replication study.

To increase statistical power, we were granted access to the results of the most recent meta-analysis reported by the DIAGRAM consortium [37], and performed a meta-analysis combining data from our Danish study with data from DIAGRAM+. This increased the total sample size to 11,729 type 2 diabetic patients and 43,943 non-diabetic individuals. In this meta-analysis, six of the ten SNPs showed nominal association with type 2 diabetes (Table 2), including the minor alleles of UQCRC1 rs2228561 (OR 1.08 [95\% CI $1.02,1.14] ; p=0.012$ ), COX5B rs 11904110 (OR 1.08 [95\% CI $1.00,1.16] ; p=0.041$ ), COX6B1 rs 2267584 (OR 0.94 [95\% CI 0.91, 0.98]; $p=0.0060$ ), COX10 rs10775377 (OR 1.05 [95\% CI 1.01, 1.09]; $p=0.0080$ ), COX10 rs8077302 (OR 0.96 [95\% CI 0.93, 1.00]; $p=0.048$ ) and COX10 rs9915302 (OR 1.07 [95\% CI 1.02, 1.12]; $p=0.0068$ ). A test of homogeneity revealed heterogeneity for $C O X 5 B$ rs1466100 $\left(I^{2}=89.2 \%, p=0.0023\right)$; for the other nine SNPs there was no significant heterogeneity between the study groups $(p>0.05)$. After adjusting for multiple testing, none of the ten SNPs was significantly associated with type 2 diabetes.

The ten SNPs found to be nominally associated with type 2 diabetes in the DIAGRAM Study [28] were investigated for potential association with quantitative diabetes-related traits in 5,869 non-diabetic participants of the population-based Inter99 cohort (ESM Tables 5-14). The minor A allele of UQCRC1 rs2228561 showed nominal association with decreased BIGTT-AIR $(\beta=-0.03, p=0.04)$, but also with increased BIGTT-S $(\beta=0.32 \%, p=0.0074)$, indicating a negative effect on glucose-stimulated insulin secretion and a positive effect on insulin sensitivity. The minor $G$ allele of COX10 rs10521253 was nominally associated with higher plasma glucose at $30 \mathrm{~min}$ after an OGTT $(\beta=0.02 \%$, $p=0.027$ ) and with decreased values of several indices of beta cell function, including the insulinogenic index $(\beta=-0.05 \%, p=0.046)$, CIR $(\beta=-0.07 \%, p=0.011)$, BIGTTAIR $(\beta=-0.04 \%, p=0.028)$ and DI $(\beta=-0.05 \%, p=0.022)$. The minor A allele of COX10 rs8077302 showed nominal association with lower fasting serum insulin $(\beta=-0.02 \%$, $p=0.049$ ), and borderline associations with decreased HOMA-IR $(\beta=-0.02 \%, p=0.054)$ and increased ISI ( $\beta=0.02 \%, p=0.054)$, all indicating a potential positive effect on insulin sensitivity. Carriers of the minor $\mathrm{C}$ allele of COX4I1 rs16939766 had lower serum insulin $120 \mathrm{~min}$ after an OGTT $(\beta=-0.06 \%, p=0.036)$, and carriers of the minor T allele in NDUFV3 rs8134542 showed lower plasma glucose $120 \mathrm{~min}$ after an OGTT $(\beta=-0.01 \%, p=0.024)$, both

Table 1 Association between common variants in or near OxPhos genes and type 2 diabetes in case-control analysis of 3,599 Danish type 2 diabetic cases and 4,956 glucose-tolerant controls

\begin{tabular}{|c|c|c|c|c|c|c|c|c|c|c|c|}
\hline \multicolumn{4}{|l|}{ Gene } & \multicolumn{3}{|c|}{ Zygosity type 2 diabetes } & \multicolumn{3}{|c|}{ Zygosity controls } & \multicolumn{2}{|l|}{ Values } \\
\hline Gene & SNP & Alleles ${ }^{\mathrm{a}}$ & MAF & Wild-type & Heterozygous & Homozygous & Wild-type & Heterozygous & Homozygous & OR $(95 \% \mathrm{CI})$ & $p$ value $^{\mathrm{b}}$ \\
\hline$C O X 5 B$ & rs11904110 & $\mathrm{T} / \mathrm{C}$ & 0.058 & 3,071 & 403 & 13 & 4,309 & 550 & 18 & $1.16(0.96,1.40)$ & 0.11 \\
\hline COX5B & rs 1466100 & $\mathrm{~A} / \mathrm{T}$ & 0.016 & 3,367 & 130 & 2 & 4,719 & 144 & 2 & $1.62(1.16,2.28)$ & 0.0052 \\
\hline UQCRC1 & rs 2228561 & $\mathrm{C} / \mathrm{T}$ & 0.13 & 2,600 & 828 & 65 & 3,661 & 1,103 & 78 & $1.12(0.98,1.28)$ & 0.084 \\
\hline COX4I1 & rs16939766 & $\mathrm{G} / \mathrm{C}$ & 0.054 & 3,112 & 381 & 9 & 4,354 & 494 & 17 & $1.08(0.89,1.3)$ & 0.44 \\
\hline COX10 & rs 10775377 & $\mathrm{~A} / \mathrm{C}$ & 0.41 & 1,189 & 1,688 & 618 & 1,727 & 2,307 & 817 & $1.07(0.98,1.17)$ & 0.14 \\
\hline COX10 & rs 10521253 & $\mathrm{~A} / \mathrm{G}$ & 0.069 & 3,033 & 439 & 10 & 4,220 & 609 & 18 & $1.01(0.84,1.17)$ & 0.94 \\
\hline COX10 & rs 8077302 & $\mathrm{G} / \mathrm{A}$ & 0.50 & 890 & 1,756 & 845 & 1,232 & 2,394 & 1,240 & $0.99(0.91,1.08)$ & 0.88 \\
\hline COX10 & rs9915302 & $\mathrm{T} / \mathrm{G}$ & 0.19 & 2,218 & 1,131 & 140 & 3,238 & 1,423 & 166 & $1.14(1.02,1.28)$ & 0.020 \\
\hline$C O X 6 B 1$ & rs 2267584 & $\mathrm{G} / \mathrm{A}$ & 0.32 & 1,657 & 1,507 & 346 & 2,226 & 2,114 & 522 & $0.93(0.85,1.02)$ & 0.13 \\
\hline NDUFV3 & rs8134542 & $\mathrm{C} / \mathrm{T}$ & 0.24 & 2,004 & 1,294 & 202 & 2,775 & 1,782 & 297 & $0.98(0.89,1.09)$ & 0.75 \\
\hline
\end{tabular}

Effect of genotype was calculated for the minor allele of each SNP

${ }^{\text {a }}$ Major/minor allele

${ }^{\mathrm{b}}$ Calculated assuming an additive model with adjustment for sex, age and BMI 
Table 2 Meta-analysis of association between common variants in or near OxPhos genes and type 2 diabetes in 11,729 type 2 diabetic cases and 43,943 non-diabetic controls

\begin{tabular}{lllll}
\hline Gene & SNP & MAF & OR $(95 \%$ CI $)$ & $p$ value \\
\hline COX5B & rs11904110 & 0.058 & $1.08(1.00,1.16)$ & 0.041 \\
COX5B & rs1466100 & 0.016 & $1.01(0.87,1.17)$ & 0.91 \\
UQCRC1 & rs2228561 & 0.13 & $1.08(1.02,1.14)$ & 0.012 \\
COX4I1 & rs16939766 & 0.054 & $1.05(0.97,1.14)$ & 0.25 \\
COX10 & rs10775377 & 0.41 & $1.05(1.01,1.09)$ & 0.0080 \\
COX10 & rs10521253 & 0.069 & $0.97(0.91,1.04)$ & 0.41 \\
COX10 & rs8077302 & 0.50 & $0.96(0.93,1.00)$ & 0.048 \\
COX10 & rs9915302 & 0.19 & $1.07(1.02,1.12)$ & 0.0068 \\
COX6B1 & rs2267584 & 0.32 & $0.94(0.91,0.98)$ & 0.0060 \\
NDUFV3 & rs8134542 & 0.24 & $1.02(0.98,1.07)$ & 0.30 \\
\hline
\end{tabular}

Combined effects and $p$ values were calculated by a fixed-effect metaanalysis applying an additive genetic model

Effect of genotype was calculated for the minor allele of each SNP

indicating improved glucose homeostasis. The remaining five SNPs showed no nominal associations with any of the investigated quantitative diabetes-related traits (data not shown). After adjustment for multiple testing none of the observed nominal associations remained significant.

\section{Discussion}

Based on the substantial evidence that mitochondrial dysfunction is linked to insulin resistance and beta cell dysfunction, and is present in multiple tissues relevant to the pathogenesis of type 2 diabetes [7-22], we hypothesised that common variation in OxPhos genes contributes to an increased risk of type 2 diabetes or influences metabolic traits related to the disease. OxPhos gene variants showing nominal association with type 2 diabetes in a meta-analysis of three GWA studies that were part of the DIAGRAM Study [28] were selected for follow-up in an independent cohort of Danish individuals. In our Danish cohort, we replicated the nominal association of the minor $\mathrm{G}$ allele of COX10 rs9915302 with type 2 diabetes, while finding that the minor $\mathrm{T}$ allele of $C O X 5 B$ rs 1466100 , rather than the major A allele, was nominally associated with type 2 diabetes. Combining the genotyping results from our Danish cohort with those from DIAGRAM+ [37], sample size was increased to 11,729 type 2 diabetic cases and 43,943 controls. In this meta-analysis, none of the investigated OxPhos gene variants showed robust association with type 2 diabetes after correction for multiple testing. Moreover, quantitative traits analysis showed only nominally significant associations between five SNPs and different metabolic traits of glucose homeostasis, such as a weak negative effect of the minor alleles of UQCRC1 rs2228561 and COX10 rs10521253 on indices of glucosestimulated insulin secretion. Taken together, these data show no robust association between common variation in OxPhos genes and type 2 diabetes. Moreover, although we cannot completely rule out the possibility that some SNPs in or near OxPhos genes may contribute to impaired glucose-stimulated insulin secretion, our results provide evidence that the major role played by insulin resistance in the pathogenesis of type 2 diabetes cannot be explained by such genetic variants.

The hypothesis of a potential association between common variation in OxPhos genes and type 2 diabetes was strongly supported a priori by numerous metabolic and physiological studies [7-18]. This makes our study unique compared with replication studies and meta-analysis of previously identified type 2 diabetes risk alleles, for most of which knowledge of the underlying phenotype leading to type 2 diabetes is limited [23]. The present study is, to our knowledge, the first to replicate a nominal association between OxPhos gene variants and type 2 diabetes. Its major strength, however, is that in a sufficiently large metaanalysis of 11,729 type 2 diabetic cases and 43,943 controls, we provide evidence that common variation in OxPhos genes does not robustly contribute to the risk of type 2 diabetes. Consistently, our analysis of quantitative metabolic traits in a Danish non-diabetic cohort demonstrated that among the OxPhos alleles showing nominal association with type 2 diabetes in the DIAGRAM study [28], none negatively influenced surrogate markers of insulin sensitivity. These results support recent observations in insulin receptor-deficient individuals showing that mitochondrial dysfunction in skeletal muscle is probably the consequence rather than the cause of insulin resistance in common forms of type 2 diabetes [38]. Thus in individuals with a heterozygous mutation in the tyrosine kinase domain of INSR, which is known to cause insulin resistance via impaired insulin signalling [39], a measure of skeletal muscle mitochondrial function in vivo was significantly reduced [38].

In our quantitative metabolic traits analysis, we observed a nominal significant negative influence of the minor alleles of UQCRC1 rs2228561 and COX10 rs10521253 on indices of glucose-stimulated insulin secretion. Correspondingly, a recent study showed two other common variants in or near OxPhos genes to be nominally associated with a decreased insulinogenic index [40]. In this study of Olsson et al. [40], variants in or near 76 nuclear-encoded OxPhos genes were explored for potential association with type 2 diabetes and insulinogenic index in the Diabetes Genetic Initiative (DGI) GWA Study [41]. In contrast to our study, the investigators were unable to find any variants nominally associated with type 2 diabetes, probably because the DGI study cohort size 
was only one third of the DIAGRAM cohort size [28]. On the other hand, Olsson et al. found nine SNPs in or near OxPhos genes showing nominal association $(p<0.01)$ with the insulinogenic index in 1,467 non-diabetic participants of the DGI study [40]. The top two of these SNPs, rs606164 adjacent to NDUFC2 and rs1323070 adjacent to COX7A2, were genotyped and nominal association replicated in a larger cohort of 4,323 non-diabetic participants from the PPP-Botnia study [40]. Because no SNPs in or near NDUFC2 or $C O X 7 A 2$ showed nominal association with type 2 diabetes in the DIAGRAM study [28], they were not genotyped for quantitative trait analysis in our 5,869 non-diabetic individuals. The study of Olsson et al. [40] provides evidence that some common variants in or near OxPhos genes may increase susceptibility to impaired beta cell function in non-diabetic individuals, even when the same OxPhos variants show no association with type 2 diabetes. Although this possibility is not supported by a very recent large-scale study of the combined effect of several OxPhos variants [42], we cannot rule out the possibility that additional OxPhos gene variants among those genotyped in the DIAGRAM study [28] would have shown association with indices of beta cell secretion in our Danish cohort.

Among the OxPhos gene variants nominally associated with type 2 diabetes in the DIAGRAM study [28], most $(\sim 90 \%)$ were found in or near nuclear-encoded genes (COX4I1, COX5B, COX6B1, COX10, COX15) involved in the regulation or assembly of COX, complex IV of the ETC. More than $70 \%$ of these COX-related SNPs were localised to COX10, and common variation in $C O X 10$ was covered by genotyping four COX10 SNPs. The nominal association of the minor $\mathrm{G}$ allele of $C O X 10$ rs9915302 with type 2 diabetes was replicated in our Danish case-control cohort, and the minor allele of COX10 rs10521253 showed nominal association with several indices of impaired beta cell function, including BIGTT-AIR, CIR, DI and insulinogenic index. The COX10 gene encodes a COX assembly protein involved in the mitochondrial haem biosynthetic pathway, and is one of six nuclear genes in which mutations cause isolated COX deficiency [43]. Although none of the examined COX-related SNPs showed robust association with type 2 diabetes, the observed enrichment of $\mathrm{COX} 10$ SNPs among variants nominally associated with type 2 diabetes suggests that sequencing of low-frequency variants in or near $C O X 10$, for example, could lead to the discovery of SNPs with a higher impact on indices of beta cell function and type 2 diabetes risk.

A potential limitation of the present study is that, based on the available evidence from in vivo studies of human skeletal muscle and beta cell islets, we decided to focus selectively on nuclear-encoded OxPhos genes. That is, we did not include transcriptional regulators of mitochondrial genes or any other of the $\sim 1,000$ genes encoding proteins proposed to reside and function within mitochondria [44]. Thus common variation in genes involved, for example, in citric acid cycle, beta-oxidation, reactive oxygen species (ROS) production and apoptosis were not studied. Moreover, we only investigated nuclear-encoded OxPhos genes, excluding any variation in the 13 OxPhos genes known to be encoded by mitochondrial DNA. However, it was recently demonstrated that common variation in genes encoded by mitochondrial DNA is not associated with type 2 diabetes or related metabolic traits [45]. Another limitation of the present study is that the studies of quantitative metabolic traits related to type 2 diabetes were based on OGTT-derived measures of insulin secretion and insulin sensitivity, rather than the application of gold-standard methods. A recent report indicates that, in particular, caution is required when interpreting differences in OGTT-derived values of insulin sensitivity, because such differences may reflect variations in beta cell function, rather than true variations in insulin sensitivity [46]. Moreover, we did not test whether the combined effect of all genotyped SNPs in or near OxPhos genes contributed to an increased risk of type 2 diabetes or negatively influenced the quantitative traits of insulin sensitivity and beta cell function. However, in a very recent study [42], which was published after the genotyping of OxPhos gene variants in our Danish individuals [47], a gene set enrichment analysis approach called MAGENTA was applied to GWA data. The results show that the combined effects of common genetic variants in transcriptional regulators of mitochondrial genes, in OxPhos genes and in all other known $(\sim 1,000)$ autosomal human mitochondrial genes were not enriched for associations with type 2 diabetes or related glycaemic traits. In the above-named study, the statistical analysis was based on $p$ values rather than genotyping results, as the latter were not available [42]. Thus these data are consistent with our meta-analysis of genotyping results from DIAGRAM+ [37] and the Danish case-control cohort showing no robust association of OxPhos gene variants with type 2 diabetes.

In summary, we report that among common variants in or near OxPhos genes potentially contributing to type 2 diabetes susceptibility, minor alleles in the COX5B and COX10 genes show only weak and nominally significant associations with type 2 diabetes in a cohort of Danish individuals. However, analysis of quantitative metabolic traits in non-diabetic participants, and a large meta-analysis of 11,729 cases and 43,943 controls including our Danish cohort provide evidence that common variation in proximity to OxPhos genes is not a major cause of insulin resistance or common forms of type 2 diabetes. 
Acknowledgements The Novo Nordisk Foundation Center for Basic Metabolic Research is funded by the Novo Nordisk Foundation. We thank the Inter99, ADDITION and Steno cohort study groups. The Inter99 Study was initiated by T. Jørgensen (principal investigator), K. Borch-Johnsen (co-principal investigator), H. Ibsen and T.F. Thomsen. The Steering Committee comprises the former two and C. Pisinger. We also thank M. McCarthy and the DIAGRAM consortium for their collaboration on the meta-analysis. The study was supported by grants from the Lundbeck Foundation (www.lucamp.org), the Danish Medical Research Council and the Novo Nordisk Foundation, including the Excellence Project 2009 grant.

Contribution statement LSS, NG, JEH, TH and $\mathrm{KH}$ were responsible for the conception and design, analysis and interpretation of data, drafting the article, revising it critically for important intellectual content and final approval of the version to be published. MW, MV and TS were responsible for analysis and interpretation of data, revising the article critically for important intellectual content and final approval of the version to be published. TJ, TL, HBN and OP were responsible for conception and design, revising the article critically for important intellectual content and final approval of the version to be published.

Duality of interest N. Grarup, T. Hansen and O. Pedersen hold personal shares in Novo Nordisk. Otherwise, the authors declare that there is no duality of interest associated with this manuscript.

\section{References}

1. Højlund K, Beck-Nielsen H (2006) Impaired glycogen synthase activity and mitochondrial dysfunction in skeletal muscle. Markers or mediators of insulin resistance in type 2 diabetes. Curr Diabetes Rev 2:375-395

2. Krebs M, Roden M (2005) Molecular mechanisms of lipidinduced insulin resistance in muscle, liver and vasculature. Diabetes Obes Metab 7:621-632

3. Cusi K, Maezono K, Osman A et al (2000) Insulin resistance differentially affects the PI 3-kinase- and MAP kinase-mediated signaling in human muscle. J Clin Invest 105:311-320

4. Højlund K, Birk JB, Klein DK et al (2009) Dysregulation of glycogen synthase $\mathrm{COOH}$ - and $\mathrm{NH}_{2}$-terminal phosphorylation by insulin in obesity and type 2 diabetes mellitus. J Clin Endocrinol Metab 94:4547-4556

5. Vind BF, Pehmøller C, Treebak JT et al (2011) Impaired insulininduced site-specific phosphorylation of TBC1 domain family, member 4 (TBC1D4) in skeletal muscle of type 2 diabetes patients is restored by endurance exercise-training. Diabetologia $54: 157-167$

6. Karlsson HK, Zierath JR, Kane S, Krook A, Lienhard GE, Wallberg-Henriksson H (2005) Insulin-stimulated phosphorylation of the Akt substrate AS160 is impaired in skeletal muscle of type 2 diabetic subjects. Diabetes 54:1692-1697

7. Højlund K, Mogensen M, Sahlin K, Beck-Nielsen H (2008) Mitochondrial dysfunction in diabetes and obesity. Endocrinol Metab Clin North Am 37:713-731

8. Patti ME, Butte AJ, Crunkhorn S et al (2003) Coordinated reduction of genes of oxidative metabolism in humans with insulin resistance and diabetes: potential role of PGC1 and NRF1. Proc Natl Acad Sci USA 100:8466-8471

9. Højlund K, Yi Z, Lefort N et al (2010) Human ATP synthase beta is phosphorylated at multiple sites and shows abnormal phosphorylation at specific sites in insulin-resistant muscle. Diabetologia 53:541-551
10. Skov V, Glintborg D, Knudsen S et al (2007) Reduced expression of nuclear-encoded genes involved in mitochondrial oxidative metabolism in skeletal muscle of insulin-resistant women with polycystic ovary syndrome. Diabetes 56:2349-2355

11. Hwang H, Bowen BP, Lefort N et al (2010) Proteomics analysis of human skeletal muscle reveals novel abnormalities in obesity and type 2 diabetes. Diabetes 59:33-42

12. Mogensen M, Sahlin K, Fernström M et al (2007) Mitochondrial respiration is decreased in skeletal muscle of patients with type 2 diabetes. Diabetes 56:1592-1599

13. Phielix E, Schrauwen-Hinderling VB, Mensink M et al (2008) Lower intrinsic ADP-stimulated mitochondrial respiration underlies in vivo mitochondrial dysfunction in muscle of male type 2 diabetic patients. Diabetes 57:2943-2949

14. Szendroedi J, Schmid AI, Chmelik M et al (2007) Muscle mitochondrial ATP synthesis and glucose transport/phosphorylation in type 2 diabetes. PLoS Med 4:e154

15. Lowell BB, Shulman GI (2005) Mitochondrial dysfunction and type 2 diabetes. Science 307:384-387

16. Dahlman I, Forsgren M, Sjögren A et al (2006) Downregulation of electron transport chain genes in visceral adipose tissue in type 2 diabetes independent of obesity and possibly involving tumor necrosis factor-alpha. Diabetes 55:1792-1799

17. Bugger H, Abel ED (2010) Mitochondria in the diabetic heart. Cardiovasc Res 88:229-240

18. Schmid AI, Szendroedi J, Chmelik M, Krssák M, Moser E, Roden M (2011) Liver ATP synthesis is lower and relates to insulin sensitivity in patients with type 2 diabetes. Diabetes Care $34: 448-453$

19. Lu H, Koshkin V, Allister EM, Gyulkhandanyan AV, Wheeler MB (2010) Molecular and metabolic evidence for mitochondrial defects associated with beta-cell dysfunction in a mouse model of type 2 diabetes. Diabetes 59:448-459

20. Anello M, Lupi R, Spampinato D et al (2005) Functional and morphological alterations of mitochondria in pancreatic beta cells from type 2 diabetic patients. Diabetologia 48:282-289

21. Koeck T, Olsson AH, Nitert MD et al (2011) A common variant in TFB1M is associated with reduced insulin secretion and increased future risk of type 2 diabetes. Cell Metab 13:80-91

22. Mulder H, Ling C (2009) Mitochondrial dysfunction in pancreatic beta-cells in type 2 diabetes. Mol Cell Endocrinol 297:34-40

23. Grarup N, Sparsø T, Hansen T (2010) Physiologic characterization of type 2 diabetes-related loci. Curr Diab Rep 10:485-497

24. Rung J, Cauchi S, Albrechtsen A et al (2009) Genetic variant near IRS1 is associated with type 2 diabetes, insulin resistance and hyperinsulinemia. Nat Genet 41:1110-1115

25. Rönn T, Poulsen P, Tuomi T et al (2009) Genetic variation in ATP5O is associated with skeletal muscle ATP50 mRNA expression and glucose uptake in young twins. PLoS One 4: e4793

26. Rönn T, Poulsen P, Hansson O et al (2008) Age influences DNA methylation and gene expression of COX7A1 in human skeletal muscle. Diabetologia 51:1159-1168

27. Ling C, Poulsen P, Simonsson S et al (2007) Genetic and epigenetic factors are associated with expression of respiratory chain component NDUFB6 in human skeletal muscle. J Clin Invest 117:3427-3435

28. Zeggini E, Scott LJ, Saxena R et al (2008) Meta-analysis of genome-wide association data and large-scale replication identifies additional susceptibility loci for type 2 diabetes. Nat Genet 40:638-645

29. Lefort N, Glancy B, Bowen B et al (2010) Increased reactive oxygen species production and lower abundance of complex I subunits and carnitine palmitoyltransferase 1B protein despite normal mitochondrial respiration in insulin-resistant human skeletal muscle. Diabetes 59:2444-2452 
30. Glumer C, Jorgensen T, Borch-Johnsen K (2003) Prevalences of diabetes and impaired glucose regulation in a Danish population: the Inter99 Study. Diabetes Care 26:2335-2340

31. Jorgensen T, Borch-Johnsen K, Thomsen TF, Ibsen H, Glumer C, Pisinger C (2003) A randomized non-pharmacological intervention study for prevention of ischaemic heart disease: baseline results Inter99. Eur J Cardiovasc Prev Rehabil 10:377-386

32. Lauritzen T, Griffin S, Borch-Johnsen K, Wareham NJ, Wolffenbuttel BH, Rutten G (2000) The ADDITION Study: proposed trial of the cost-effectiveness of an intensive multifactorial intervention on morbidity and mortality among people with type 2 diabetes detected by screening. Int $\mathrm{J}$ Obes Relat Metab Disord 24(Suppl 3):S6-S11

33. World Health Organization (WHO) (1999) World Health Organization Diagnosis and Classification of Diabetes Mellitus: Report of a WHO Consultation. Part 1. WHO, Geneva

34. Grarup N, Andersen G, Krarup NT et al (2008) Association testing of novel type 2 diabetes risk alleles in the JAZF1, CDC123/CAMK1D, TSPAN8, THADA, ADAMTS9, and NOTCH2 loci with insulin release, insulin sensitivity, and obesity in a population-based sample of 4,516 glucose-tolerant middle-aged Danes. Diabetes 57:2534-2540

35. Matthews DR, Hosker JP, Rudenski AS, Naylor BA, Treacher DF, Turner RC (1985) Homeostasis model assessment: insulin resistance and beta-cell function from fasting plasma glucose and insulin concentrations in man. Diabetologia 28:412-419

36. Hansen T, Drivsholm T, Urhammer SA et al (2007) The BIGTT test: a novel test for simultaneous measurement of pancreatic beta-cell function, insulin sensitivity, and glucose tolerance. Diabetes Care 30:257-262

37. Voight BF, Scott LJ, Steinthorsdottir V et al (2010) Twelve type 2 diabetes susceptibility loci identified through large-scale association analysis. Nat Genet 42:579-589
38. Sleigh A, Raymond-Barker P, Thackray K et al (2011) Mitochondrial dysfunction in patients with primary congenital insulin resistance. J Clin Invest 121:2457-2461

39. Højlund K, Wojtaszewski JF, Birk J, Hansen BF, Vestergaard H, Beck-Nielsen H (2006) Partial rescue of in vivo insulin signalling in skeletal muscle by impaired insulin clearance in heterozygous carriers of a mutation in the insulin receptor gene. Diabetologia 49:1827-1837

40. Olsson AH, Rönn T, Ladenvall C et al (2011) Two common genetic variants near nuclear-encoded OXPHOS genes are associated with insulin secretion in vivo. Eur J Endocrinol 164:765-771

41. Saxena R, Voight BF, Lyssenko V et al (2007) Genome-wide association analysis identifies loci for type 2 diabetes and triglyceride levels. Science 316:1331-1336

42. Segrè AV, DIAGRAM Consortium; MAGIC investigators et al (2010) Common inherited variation in mitochondrial genes is not enriched for associations with type 2 diabetes or related glycemic traits. PLoS Genet 6:e1001058

43. Antonicka H, Leary SC, Guercin GH et al (2003) Mutations in COX10 result in a defect in mitochondrial heme $A$ biosynthesis and account for multiple, early-onset clinical phenotypes associated with isolated COX deficiency. Hum Mol Genet 12:2693-2702

44. Pagliarini DJ, Calvo SE, Chang B et al (2008) A mitochondrial protein compendium elucidates complex I disease biology. Cell 134:112-123

45. Saxena R, de Bakker PI, Singer K et al (2006) Comprehensive association testing of common mitochondrial DNA variation in metabolic disease. Am J Hum Genet 79:54-61

46. Hücking K, Watanabe RM, Stefanovski D, Bergman RN (2008) OGTT-derived measures of insulin sensitivity are confounded by factors other than insulin sensitivity itself. Obesity (Silver Spring) 16:1938-1945

47. Snogdal LS, Wod M, Vestmar MA et al (2010) Genetic variants involved in mitochondrial oxidative metabolism are associated with type 2 diabetes mellitus in studies of 8,441 Danes. Diabetes 59(Suppl 1):A331 (Abstract) 